\title{
Studies on Chitin XVI. Influence of Controlled Side Chain Introduction to Chitosan on the Adsorption of Copper(II) Ion
}

\author{
Keisuke KurITA, Yoshiyuki Koyama, and Satoyuki CHIKAOKA \\ Department of Industrial Chemistry, Faculty of Engineering, \\ Seikei University, Musashino-shi, Tokyo 180, Japan
}

(Received April 12, 1988)

\begin{abstract}
N-Nonanoylation of chitosan was studied in comparison with $\mathrm{N}$-acetylation, and the influence of modifications on the adsorption of copper(II) ion was elucidated to develop high performance adsorbents based on this under-utilized abundant resource. Controlled acylations with the corresponding acyl chlorides were established in a highly swelled state. The adsorption capacities proved to be highly enhanced at low extents of substitution; more remarkable improvement was achieved with nonanoyl groups than acetyl groups.

KEY WORDS Chitosan / Chemical Modification / Nonanoylation / Acetylation / Metal Cation Adsorption / Chelating Polymer /
\end{abstract}

Chitin is a readily accessible but almost unutilized resource present in large quantities and thus attracting much attention recently. Although many possibilities of utilization have been discussed so far on the basis of its unique structure and properties, high performance adsorbents seem to be promising on account of the presence of amino groups. ${ }^{1}$ Adsorbents derived from naturally occurring polymers are advantageous in view of environmental protection. Furthermore, among many kinds of chelating polymers of natural origin, chitosan has been considered to be the most powerful adsorbent for metal cations. ${ }^{2}$

In the course of the study on the effects of chemical modifications of chitin on the adsorption behavior, water-soluble partially deacetylated chitin was found to be superior to highly deacetylated chitosan. ${ }^{3}$ Moreover, loose crosslinking was found quite effective to further enhance the capacity. ${ }^{4,5}$

These results are interpreted in terms of the interference of the tight arrangement of the rigid polysaccharide molecules as a result of moderate extents of random modifications leading to increased hydrophilicity and easy accessibility of coordinating groups. This suggests the possibility of bringing about such effects through the introduction of appropriate simple substituents which enables more quantitative analysis than crosslinking. Acyl groups, especially relatively long ones, are anticipated to play a similar role to that of the bridges by loose crosslinking, and some preliminary experiments have shown the introduction of substituents to chitosan to potentially improve the properties. ${ }^{6}$ We report here the results of a detailed study on the introduction of nonanoyl groups, $\mathrm{CH}_{3}\left(\mathrm{CH}_{2}\right)_{7} \mathrm{CO}-$, and also the influence on adsorption behavior in comparison with those of acetyl groups.

\section{EXPERIMENTAL}

Chitosan was prepared by treating pulverized chitin obtained from shrimp shells (Penaeus japonicus) with $50 \%$ aqueous sodium hydroxide under nitrogen at $135^{\circ} \mathrm{C}$ for $3.5 \mathrm{~h}$. 
The degree of deacetylation was 0.88 as determined by IR spectroscopy. ${ }^{7} \mathrm{X}$-Ray diffraction diagrams were taken by the powder method with the use of nickel-filtered $\mathrm{Cu} K_{\alpha}$ radiation.

\section{Nonanoylation of Chitosan}

Chitosan, $0.4 \mathrm{~g}$, was dissolved in $10 \mathrm{~cm}^{3}$ of $10 \%$ aqueous acetic acid, and the solution was diluted with $10 \mathrm{~cm}^{3}$ of methanol. It was poured into $60 \mathrm{~cm}^{3}$ of pyridine to give a highly swelled precipitate of chitosan. The mixture was centrifuged, and $60 \mathrm{~cm}^{3}$ of the supernatant was replaced by the same amount of pyridine. This solvent replacement procedure was repeated five times. A given amount of nonanoyl chloride was added dropwise at $0^{\circ} \mathrm{C}$ with stirring, and the mixture was allowed to warm to room temperature. After $3 \mathrm{~h}$, the mixture was poured into $500 \mathrm{~cm}^{3}$ of acetone/water $(7: 1)$. The precipitate was collected on a filter, washed with acetone and then with methanol. Without drying, it was added to $80 \mathrm{~cm}^{3}$ of $1 \mathrm{~mol}^{-1}$ methanolic potassium hydroxide, and the mixture was stirred at room temperature for $24 \mathrm{~h}$. The precipitate was washed with methanol, pulverized, extracted with methanol using a Soxhlet extractor for $14 \mathrm{~h}$, and dried.

Acetylated chitosans were prepared by the same method.

\section{Adsorption of Copper(II) Ion by Acyl Chitosans}

To $25 \mathrm{~cm}^{3}$ of $5.00 \times 10^{-4} \mathrm{moll}^{-1}$ aqueous copper(II) chloride solution ( $\mathrm{pH}$ 5.2) was added $50 \mathrm{mg}$ of a chitosan derivative $(100-200$ mesh), and the mixture was stirred at room temperature for $72 \mathrm{~h}$. It was then centrifuged and filtered. The content of copper ion in the filtrate was determined by UV spectroscopy using sodium diethyldithiocarbamate. ${ }^{4}$

\section{RESULTS AND DISCUSSION}

\section{Preparation of Nonanoylated and Acetylated Chitosans}

Loose crosslinking of water-soluble chitin ${ }^{4}$ and chitosan ${ }^{5}$ with glutaraldehyde under homogeneous conditions caused random substitution along the backbone, resulting in enhanced adsorption capacity. At a high crosslinking density, the capacity decreased again, and thus controlled modifications of appropriate types are necessary to develop adsorbents of improved properties. Nonanoyl groups were thus introduced randomly to chitosan. Although acid anhydrides are most commonly used for the acylation of polysaccharides including chitin with some exceptions, ${ }^{8-13}$ it is considered useful if controlled acylations can be carried out with more easily available but less stable acyl chlorides.

The reaction was conducted in a highly swelled state to enable random substitution along the backbones for effective destruction of the crystalline structure. A highly swelled chitosan precipitate was prepared by pouring a chitosan solution into pyridine. The resulting mixture contained large amounts of protic components which interfered with the reaction, and thus the solvent was replaced repeatedly by fresh pyridine to ensure efficient acylation. ${ }^{14}$ After the reaction, the products were treated with $1 \mathrm{~mol}^{-1}$ methanolic potassium hydroxide at room temperature for $24 \mathrm{~h}$ to regenerate unreacted amino groups and also to remove ester groups whose amount was trace judging from the IR spectra. During this procedure, no reduction in the acylation degree was observed. The products were obtained as white to slightly yellow powdery materials. It is noteworthy that both the nonanoylation and acetylation proceeded quite efficiently and reproducibly when carried out in a highly swelled state under these mild conditions in spite of the reactions with acyl chlorides as summarized in Table I. The original chitosan used in this study was a sample with a degree of deacetylation of 0.88 ; namely the degree of acetylation was 0.12 . Hence, two kinds of acyl groups were present in these nonanoylated products. With the same amounts of acyl chlorides, acetyl chloride 

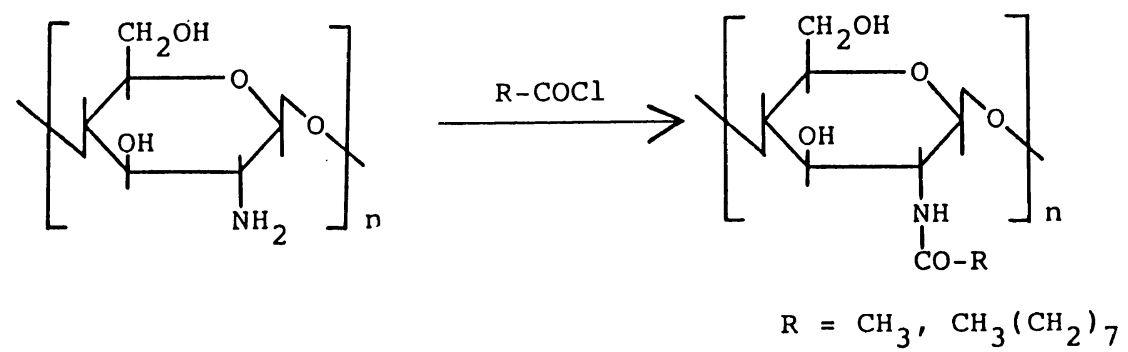

Table I. Preparation of acylated chitosans and their solubility

\begin{tabular}{|c|c|c|c|c|c|}
\hline \multirow{2}{*}{$\begin{array}{c}\text { Acyl } \\
\text { derivative }\end{array}$} & \multirow{2}{*}{$\begin{array}{l}\text { Acylating } \\
\text { reagent }^{\mathrm{a}}\end{array}$} & \multirow{2}{*}{$\begin{array}{l}\text { Reactant ratio } \\
-\mathrm{COCl} /-\mathrm{NH}_{2}\end{array}$} & \multicolumn{2}{|c|}{ Degree of substitution } & \multirow{2}{*}{ Solubility ${ }^{\mathrm{e}}$} \\
\hline & & & Acetyl $^{\mathrm{c}}$ & Nonanoyl $^{\mathrm{d}}$ & \\
\hline N-1 & $\mathrm{NC}$ & 1 & 0.12 & 0.05 & + \\
\hline $\mathbf{N}-2$ & $\mathrm{NC}$ & 2 & 0.12 & 0.10 & + \\
\hline $\mathbf{N}-3$ & $\mathrm{NC}$ & 10 & 0.12 & 0.29 & - \\
\hline$N-4$ & $\mathrm{NC}$ & 20 & 0.12 & 0.40 & - \\
\hline A-1 & $\mathrm{AC}$ & 1 & 0.16 & - & + \\
\hline A-2 & $\mathrm{AC}$ & 2 & 0.18 & - & + \\
\hline A-3 & $\mathrm{AC}$ & 5 & 0.45 & - & \pm \\
\hline$A-4$ & $\mathrm{AC}$ & 10 & 0.60 & - & - \\
\hline A-5 & $\mathrm{AC}$ & 20 & 0.71 & - & - \\
\hline
\end{tabular}

a NC, nonanoyl chloride; AC, acetyl chloride.

b Molar ratio.

c Determined by IR spectroscopy.

d Determined by the $\mathrm{C} / \mathrm{N}$ ratio of elemental analysis.

e In $5 \%$ aqueous acetic acid: + , soluble; \pm , partially soluble; - , insoluble.

gave higher substitution degrees in general than nonanoyl chloride.

The qualitative solubility of these derivatives in aqueous acetic acid was examined, and the results are included in Table I. Chitosan is soluble in aqueous acidic media, and this limits the use as an adsorbent in low $\mathrm{pH}$ regions. In both series of acyl derivatives, however, the solubility decreased with increasing extent of substitution as expected. The nonanoylated chitosans with substitution degrees of $0.05(\mathbf{N}-1)$ and $0.10(\mathbf{N}-2)$ were still soluble in aqueous acetic acid as is the original chitosan, whereas those with degrees above around 0.3 were insoluble. An acetylated chitosan with a degree of 0.45 (A-3) was still partially soluble and that of 0.60 was in- soluble, indicating nonanoyl groups to be more effective for insolubilization of chitosan owing to the higher hydrophobicity.

Figure 1 represents the IR spectra of chitosan and its acyl derivatives. The acetylated chitosans with high substitution degrees showed strong amide I and II bands at 1650 and $1550 \mathrm{~cm}^{-1}$ as shown by A-5 with a degree of 0.71 . In addition to similar characteristics regarding the amide bands, nonanoyl derivatives showed strong $\mathrm{C}-\mathrm{H}$ stretching bands, which is evident in the spectrum of a derivative with a substitution degree of $0.40(\mathbf{N}-4)$.

\section{Adsorption of Copper(II) Ion by the Chitosan Derivatives}

The adsorption behavior of the resulting 


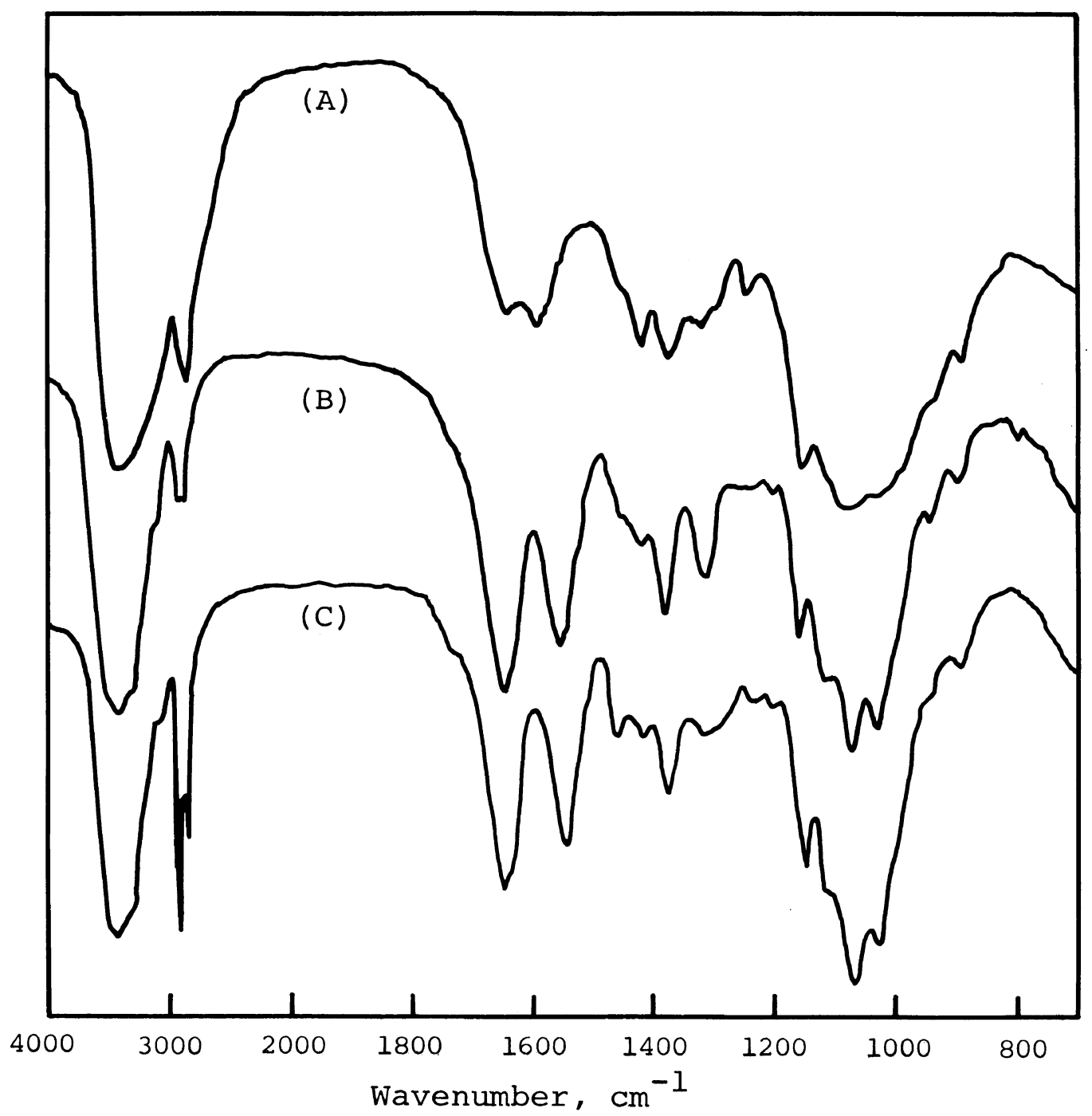

Figure 1. IR spectra (KBr) of (A) chitosan, (B) acetylated chitosan A-5, and (C) nonanoylated chitosan N-4.

nonanoylated chitosans was examined in comparison with that of the acetylated chitosans. The results are illustrated in Figure 2; the numbers on the abscissa indicate the total degree of acylations in the case of nonanoyl derivatives. The point at the lowest acylation, 0.12 , represents the capacity of the original chitosan. The adsorption capacities increase by acylation, and it is noteworthy that the profiles are quite similar to those obtained by crosslinking. ${ }^{4,5}$

The improvement of the capacity is more remarkable on nonanoylation possibly due to the more efficient interference with the tight arrangement of the chitosan molecules by the longer side chains introduced and also by the presence of the two kinds of acyl groups. The reduction in the capacity at high substitution degrees was also more pronounced with the nonanoyl derivatives owing to the longer 


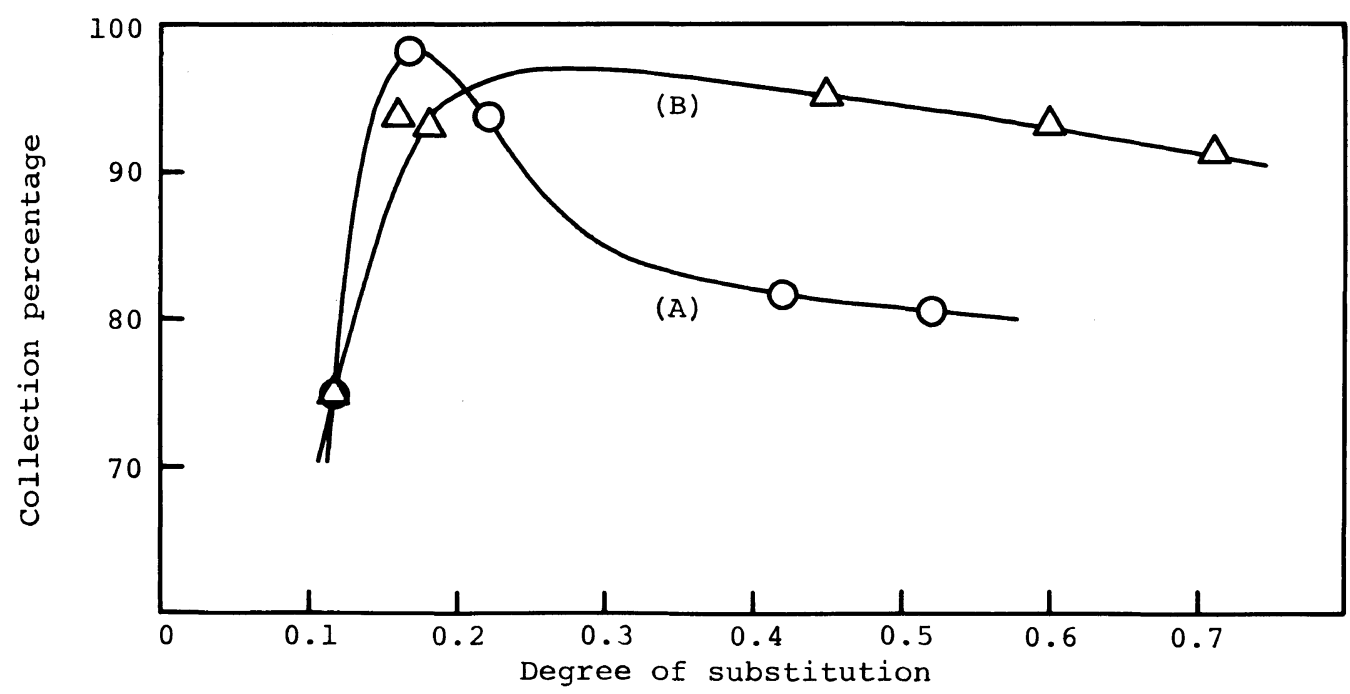

Figure 2. Adsorption of copper ion by (A) nonanoylated chitosans and (B) acetylated chitosans.

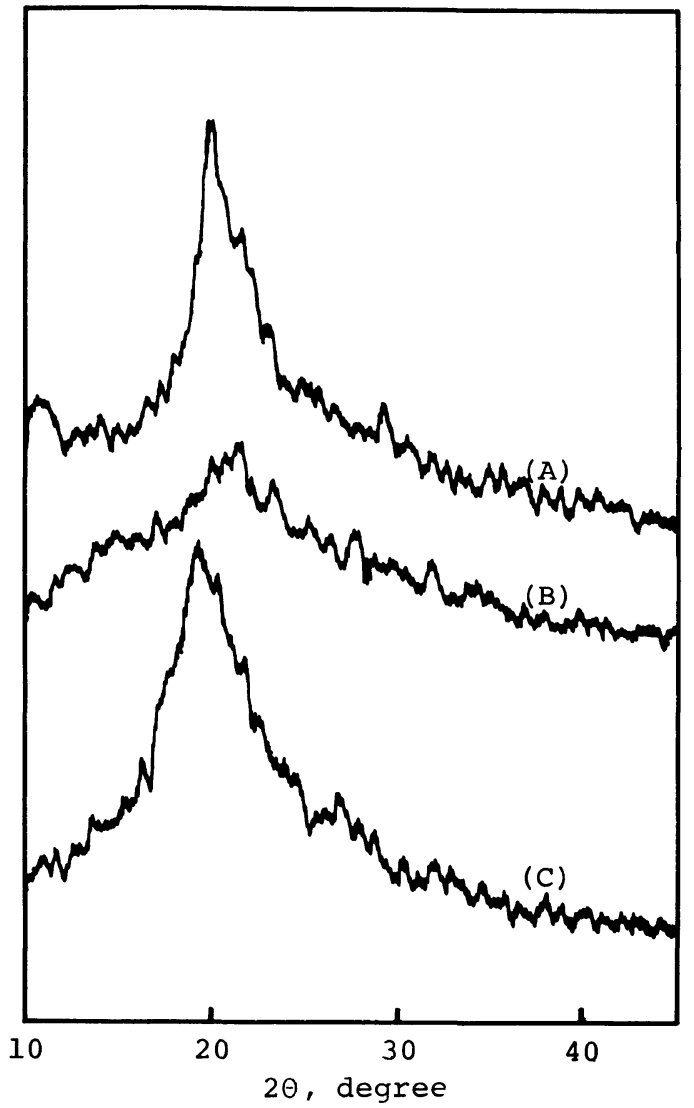

Figure 3. X-Ray diffraction diagrams of (A) chitosan, (B) nonanoylated chitosan $\mathbf{N}-1$, and (C) nonanoylated chitosan $\mathbf{N}-4$.

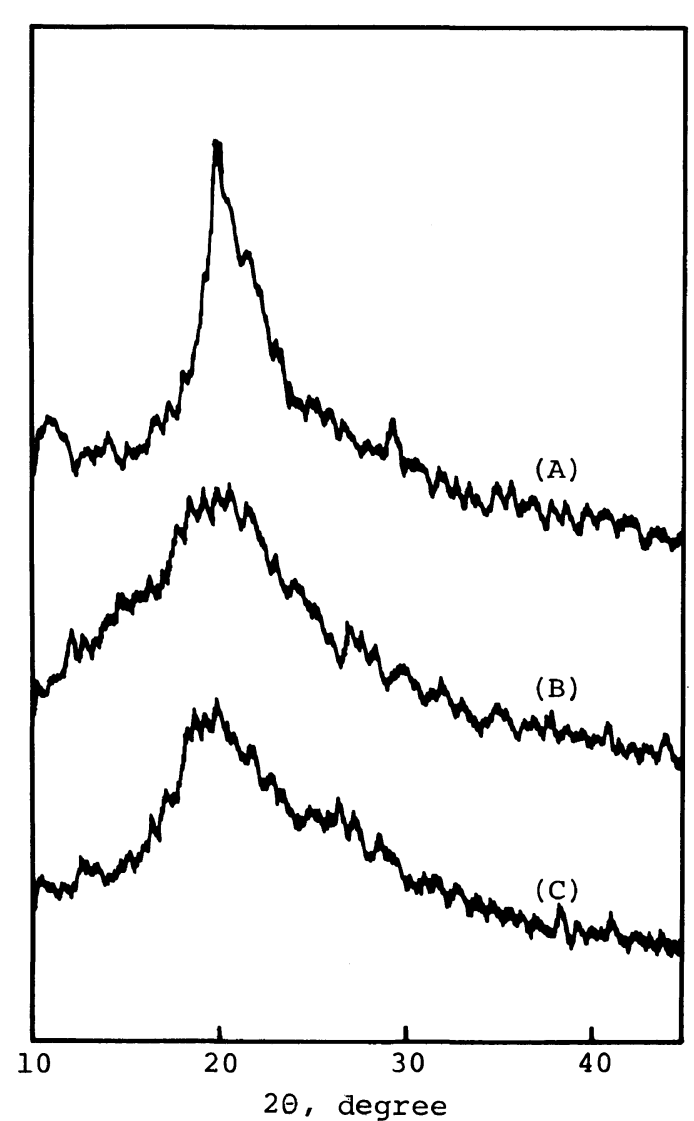

Figure 4. X-Ray diffraction diagrams of (A) chitosan, (B) acetylated chitosan A-1, and (C) acetylated chitosan A-5. 


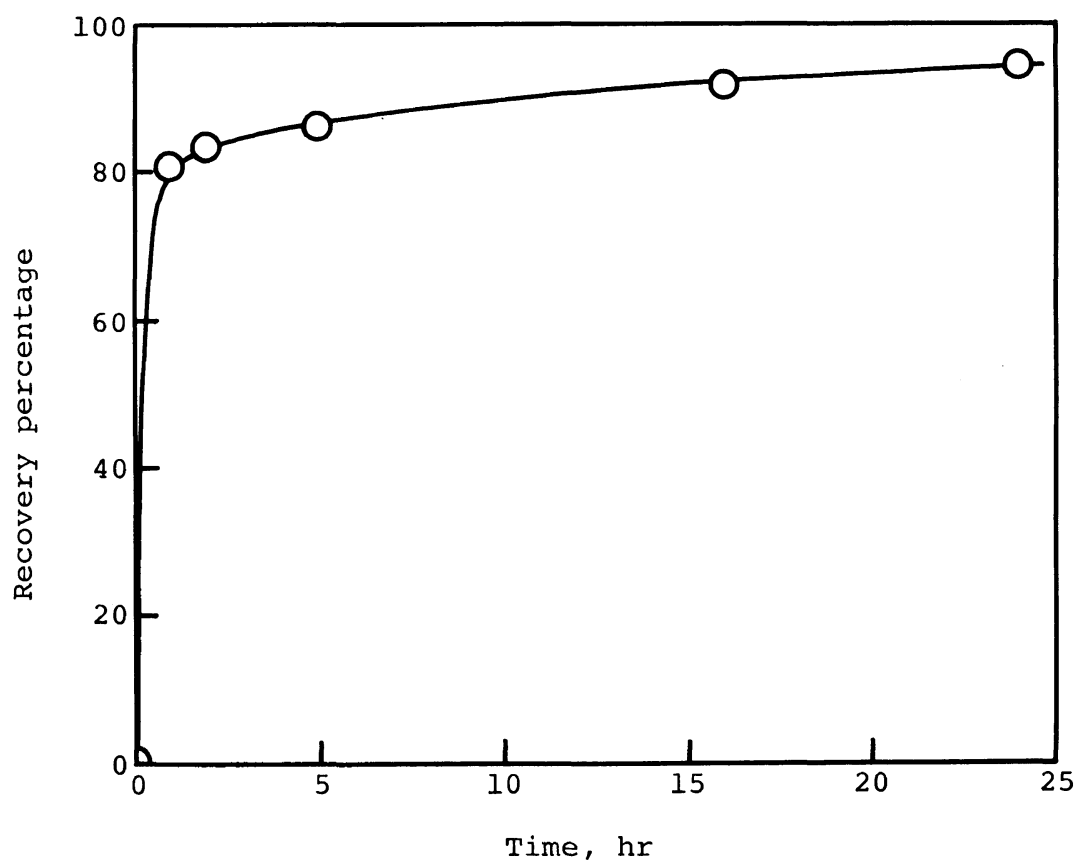

Figure 5. Desorption of copper ion from the adsorption complex of nonanoylated chitosan N-3 at pH 2.

chain. These results indicate the longer substituents to be more effective for enhancement of the capacity as well as insolubilization at relatively low substitution degrees.

\section{Crystallinity Change}

The above results imply the change in the crystallinity of chitosan on acylation, and it was examined by X-ray diffractiometry. As anticipated, only low extents of acylation were sufficient to destroy the crystalline structure. The diagrams of derivatives $\mathbf{N}-1$ and A-1 with almost the same substitution degrees indicate the nonanoylation to cause more efficient destruction of the crystalline structure (Figures 3 and 4). This is in good accordance with the more remarkable capacity enhancement by nonanoylation. At higher substitution degrees, nonanoylated chitosans assumed crystalline structures again unlike acetylated chitosans. Crystallization of highly nonanoylated chitosans reasonably explain the rather marked reduction in the adsorption capacity in the high substitution regions compared with that of acetyl derivatives.

\section{Desorption from the Adsorption Complex}

Desorption of copper ion from adsorption complexes was examined in diluted hydrochloric acid at $\mathrm{pH} 2$ where no adsorption occurred. Figure 5 shows the desorption behavior of the complex obtained from N-3. The copper ion in the complex was liberated smoothly and recovered almost quantitatively in $24 \mathrm{~h}$. Since the nonanoylated chitosan used here is insoluble in aqueous acidic solvents and can be recovered almost quantitatively from the mixture of desorption experiment, the adsorption capacity of the recovered sample was also determined. The capacity of $\mathbf{N}-3$ for the second run was $83 \%$, almost unchanged from the value $(79-82 \%)$ for the first run, showing the insoluble acylated derivatives to be suitable for repeated use.

\section{CONCLUSIONS}

Controlled introduction of acyl groups to 
chitosan was effective to produce adsorbents of improved properties, and nonanoyl groups were superior to acetyl groups in both capacity enhancement and insolubilization. These remarkable effects were especially obvious at low extents of substitution, indicating the importance of balance between the two conflicting effects brought about by acylation, a favorable effect of destruction of the molecular arrangement and an unfavorable effect of increased hydrophobicity.

Acknowledgments. This work was partly supported by the Grant-in-Aid for Scientific Research on Priority Areas, New Functional Materials-Design, Preparations, and Control-, the Ministry of Education, Science, and Culture, \#62604600, and also by the Science Research Promotion Fund, Japan Private School Promotion Foundation.

\section{REFERENCES}

1. K. Kurita, Yukigosei Kagaku Kyokaishi, 42, 567 (1984); Kobunshi, 36, 406 (1987).

2. R. A. A. Muzzarelli, "Natural Chelating Polymers," Pergamon Press, Oxford, 1973.

3. K. Kurita, T. Sannan, and Y. Iwakura, J. Appl. Polym. Sci., 23, 511 (1979).

4. K. Kurita, Y. Koyama, and A. Taniguchi, J. Appl. Polym. Sci., 31, 1169 (1986).

5. K. Kurita, Y. Koyama, and A. Taniguchi, J. Appl. Polym. Sci., 31, 1951 (1986).

6. K. Kurita, S. Chikaoka, and Y. Koyama, Chem. Lett., 9 (1988).

7. T. Sannan, K. Kurita, K. Ogura, and Y. Iwakura, Polymer, 19, 458 (1978).

8. K. Akagane and G. G. Allan, Shikizai Kyokaishi, 46, 379 (1973).

9. S. Hirano and Y. Koide, Carbohydr. Res., 65, 166 (1978).

10. O. Somorin, N. Nishi, S. Tokura, and J. Noguchi, Polym. J., 11, 391 (1979).

11. C. L. McCormick and D. K. Lichatowich, J. Polym. Sci., Polym. Lett. Ed., 17, 479 (1979).

12. S. Fujii, H. Kumagai, and M. Noda, Carbohydr. Res., 83, 389 (1980).

13. K. Kaifu, N. Nishi, and T. Komai, J. Polym. Sci., Polym. Chem. Ed., 19, 2361 (1981).

14. K. Kurita, S. Chikaoka, M. Kamiya, and Y. Koyama, Bull. Chem. Soc. Jpn., 61, 927 (1988). 